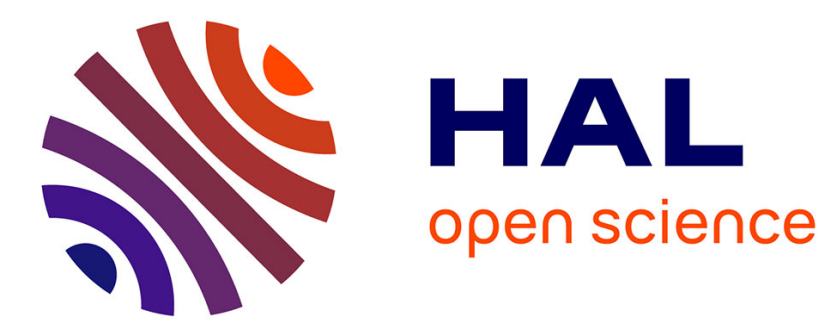

\title{
La troisième dimension de la scoliose: le plan axial oublié
}

\author{
Tamás Sándor Illés, François Lavaste, Jean Félix Dubousset
}

\section{To cite this version:}

Tamás Sándor Illés, François Lavaste, Jean Félix Dubousset. La troisième dimension de la scoliose: le plan axial oublié. Revue de Chirurgie Orthopédique et Traumatologique, 2019, 105, pp.204 - 212. 10.1016/j.rcot.2018.12.021 . hal-03486565

\section{HAL Id: hal-03486565 https://hal.science/hal-03486565}

Submitted on 20 Dec 2021

HAL is a multi-disciplinary open access archive for the deposit and dissemination of scientific research documents, whether they are published or not. The documents may come from teaching and research institutions in France or abroad, or from public or private research centers.
L'archive ouverte pluridisciplinaire HAL, est destinée au dépôt et à la diffusion de documents scientifiques de niveau recherche, publiés ou non, émanant des établissements d'enseignement et de recherche français ou étrangers, des laboratoires publics ou privés.

\section{다)(1) $(5$}

Distributed under a Creative Commons Attribution - NonCommercial| 4.0 International 


\section{Mise au point}

\section{La troisième dimension de la scoliose : le plan axial oublié The Third Dimension of Scoliosis: The Forgotten Axial Plane}

Tamás S. ILLÉS ${ }^{1,2,3}$, Francois LAVASTE ${ }^{4}$, Jean F. DUBOUSSET ${ }^{3}$,

${ }^{1}$ Département de Chirurgie Orthopédique et de Traumatologie, CHU - Brugmann

Université Libre de Bruxelles/Vrije Universiteit Brussel, Bruxelles, Belgique

${ }^{2}$ Département de Chirurgie Orthopédique et de Traumatologie, CHU - Odense et Institut de Recherche Clinique, Université du Danemark Sud, Odense, Danemark

${ }^{3}$ Académie Nationale de Médecine, Paris, France

${ }^{4}$ Institut de Biomécanique Humaine Georges Charpak Arts et Métiers ParisTech, Paris, France

Adresse de correspondance :

Prof. Dr. Tamás S. Illés

Département de Chirurgie Orthopédique et de Traumatologie,

CHU - Brugmann

Place Van Gehuchten 4.

1020 Bruxelles

Belgique

Tel: +3224773080

Fax: +32 24772161

e-mail: tamasilles58@yahoo.com

e-mail: tamas.illes@chu-brugmann.be 
Ne pas utiliser, pour citation, la référence française de cet article, mais celle de l'article original paru dans Orthopaedics \&Traumatology: Surgery \& Research, en utilisant le DOI ci-dessus.

\section{RÉSUMÉ}

La scoliose idiopathique est une déformation tridimensionnelle (3D) de la colonne vertébrale. Dans la pratique médicale, le diagnostic et le traitement de la scoliose sont bidimensionnels (2D) et uniquement basés sur des images radiographiques postéro-antérieures (PA) et latérales. Ces images permettent aux cliniciens d'analyser les projections dans seulement deux plans de la déformation et ne permettent pas d'analyser la projection dans le plan axial des courbes, ce qui fausse l'évaluation correcte d'une déformation 3D. Dans tous les domaines de l'ingénierie la norme de référence est qu'il est indispensable de créer un dessin des plans de l'objet projeté dans les trois plans de l'espace pour concevoir cet objet spatial 3D. Pour une déformation 3D, la connaissance des changements dans les trois plans est également nécessaire car le troisième plan est tout aussi important que les deux autres. Dans cette étude, nous avons résumé le développement chronologique de la visualisation du plan axial et des mesures de la déformation de la colonne vertébrale.

Mots de clés :

Scoliose, plan axial; vue de dessus, vecteur vertébral 


\section{INTRODUCTION}

La scoliose idiopathique est une déformation tridimensionnelle (3D) de la colonne vertébrale. Son diagnostic et son traitement reposent uniquement sur des images radiographiques postéro-antérieures (PA) et latérales.

Dans la pratique de l'ingénierie, la création d'un dessin projeté de l'objet dans les trois plans de l'espace est importante pour concevoir et créer un objet spatial 3D. Cette approche est la référence dans tous les domaines de l'ingénierie, y compris l'aéronautique, l'industrie automobile et le génie civil.

Contrairement à la pratique en ingénierie et malgré la nature 3D de la scoliose, en médecine, le diagnostic et le traitement de la scoliose sont bidimensionnels (2D) car les images PA et les radiographies latérales ne permettent aux cliniciens que l'analyse des projections bidimensionnelles de la déformation et ne permettent pas une analyse de la projection dans le plan axial des courbes. Cette approche a des racines historiques mais est inacceptable pour évaluer une déformation 3D, dans laquelle il est nécessaire de connaître des changements dans les trois plans de l'espace, car le troisième plan est tout aussi important que les deux autres. Dans cet article, nous avons résumé le développement chronologique de la visualisation et des mesures du plan axial et discuté des dernières tendances et des méthodes les plus prometteuses.

La nature multidimensionnelle de la scoliose est supposée depuis des siècles : à la fin des années 1700, Magny fut le premier à décrire la rotation vertébrale associée à la déformation de la colonne vertébrale (Fig. 1) [1].

Après avoir examiné plusieurs pièces anatomiques de la colonne scoliotique, Adams a très précisément décrit les rotations dans le plan axial des vertèbres de la déformation scoliotique. Il a également clairement décrit d'autres altérations du plan axial, telles que des déformations des côtes et de la cage thoracique avec des gibbosités, qui sont le résultat de la rotation axiale des vertèbres ou d'un déplacement latéral des vertèbres du à la formation d'une scoliose (Fig. 2) [2]. Compte tenu de ces analyses et descriptions anatomiques précises, la nature spatiale de la scoliose devint évidente à la fin du XIXe siècle.

Au début du XXe siècle, l'avènement de la radiographie offrit la première occasion d'évaluer directement les déformations chez les patients vivants [3]. En raison de l'utilisation généralisée des images radiographiques la plupart des chirurgiens oublièrent progressivement la nature spatiale de la scoliose, celle-ci étant principalement évaluée et mesurée à l'aide d'images du plan coronal. Actuellement l'angle de Cobb est considéré comme l'étalon-or pour quantifier la gravité d'une scoliose [4].

Malgré le caractère 3D reconnu de la scoliose, l'analyse des caractéristiques de la courbure sagittale a également une histoire relativement courte car les changements les plus visibles de la scoliose se situent dans le plan frontal. A contrario les modifications de la courbure sagittale sont difficiles à évaluer car il est également 
difficile de décrire avec précision les variations naturelles des courbures sagittales. Par conséquent, on a récemment mis l'accent sur la caractérisation des courbures sagittales normales plutôt que sur l'évaluation des composantes des déformations du plan sagittal. Il est relativement facile d'effectuer ces études car les courbes sagittales peuvent être affichées et analysées sur les clichés latéraux. Aujourd'hui, l'alignement sagittal harmonieux et les modifications des courbures sagittales associées à la scoliose sont parmi les données les plus fréquemment analysées de la colonne vertébrale [5,6]. Les mesures du plan sagittal ont même été intégrées dans un système largement utilisé de classification de la scoliose [7].

Malheureusement, ce n'est pas le cas pour l'analyse des lésions du plan axial, bien que l'auteur senior de cet article ait déjà attiré l'attention sur l'importance de la déformation de la scoliose dans le plan axial [8]. L'équipe de recherche de l'auteur senior a tenté d'analyser la projection 3D des déformations de la colonne vertébrale à l'aide de la technologie informatique. Une reconstruction spatiale idéalisée de la colonne vertébrale a été effectuée après avoir marqué les limites postérieures du plateau sacré et le centre de chaque plateau vertébral sur les images PA et le bord postérieur de chaque vertèbre sur les rayons X latéraux. De plus, la rotation vertébrale a été déterminée à chaque niveau sur des radiographies PA, en utilisant la méthode Perdriolle. Avec l'aide d'un logiciel spécial, l'ordinateur a directement produit une reconstruction 3D de la colonne vertébrale. La vue axiale du haut de la colonne vertébrale a également été visualisée, ce qui semble être la vue la plus utile (Fig. 3). Trente cas de scoliose infantile ont été analysés en utilisant cette méthode pour séparer les patients atteints de scoliose évolutive de ceux présentant une scoliose non progressive. Six groupes pronostiques différents ont été créés, soulignant le fait que ces groupes pronostiques ne pouvaient être identifiés sur la base de radiographies simples de face et de profil.

La reconnaissance de l'importance des changements du plan horizontal a conduit au développement d'une technique chirurgicale de dérotation. L'introduction de l'instrumentation Cotrel - Dubousset (ICD) et sa philosophie de traitement, qui ont permis de corriger en 3D les déformations de la colonne vertébrale, ont été la conséquence la plus importante de la reconnaissance des altérations du plan axial associées au développement de la scoliose [9].

En outre, l'identification et l'analyse des altérations du plan axial ont abouti à la reconnaissance de changements postopératoires fondamentaux, tels que le phénomène du vilebrequin après arthrodèse postérieure [10]. Dans ce phénomène, au début de la fusion postérieure, les corps vertébraux antérieurs continuent de croître, entraînant une hyper-rotation des vertèbres dans le plan axial (Fig. 4). Ce mécanisme est difficile à comprendre et peut-être même incompréhensible sans l'analyse des images de la vue de dessus [10]. La cyphose paradoxale hyperrotatoire, qui est un phénomène courant chez les patients présentant une courbure importante, ne peut être comprise, même sans analyse du plan axial [11].

Malheureusement, ces premières conclusions sur l'importance des modifications du plan axial n'ont pas été comprises. 
L'adoption mondiale de l'ICD a généré une forte demande vers l'analyse de la projection de la scoliose dans les trois plans de l'espace [12]. En 1994, le groupe de travail «3D Terminology of Spinal Deformity de la Scoliosis Research Society (SRS) a souligné la nécessité d'une méthode de diagnostic véritablement 3D permettant de diagnostiquer et d'évaluer avec précision les déformations scoliotiques dans les trois plans [13].

L'évaluation la plus précise des altérations du plan horizontal semble être réalisée par tomodensitométrie (Fig. 5) et par résonance magnétique [14-19]. Malgré le développement de ces technologies d'imagerie et leur influence sur les diagnostics, leur utilité dans l'imagerie de routine de la scoliose est limitée car elles sont associées à des doses de rayonnement et à des coûts élevés. L'effet de la position couchée pendant l'imagerie, qui peut également entraîner des altérations significatives de la courbure dans les plans coronal, sagittal et horizontal, constitue un obstacle aussi à l'utilisation de ces modalités d'imagerie dans la pratique orthopédique quotidienne [20].

Par conséquent, l'outil principal de diagnostic continue à être l'imagerie 2D, les radiographies PA et latérale. Cependant, la visualisation et l'analyse des altérations du plan axial restent un véritable défi et sont particulièrement difficiles à réaliser en pratique clinique.

\section{MESURES 2D}

Comme les rayons X 2D constituent la base de l'évaluation clinique de la scoliose, plusieurs méthodes ou calculs mathématiques furent développés pour quantifier la rotation au plan axial. Les mesures sont effectuées à partir de repères anatomiques des vertèbres, tels que les pédicules et les apophyses épineuses, visibles sur les radiographies frontale et sagittale.

Malgré la possibilité de réaliser une imagerie à rayons $\mathrm{X}$ en 2D, pendant longtemps seules les images de projection en PA furent utilisées pour déterminer la rotation axiale basée sur l'évaluation de la position relative des éléments vertébraux postérieurs. Cobb détermine la rotation en fonction de la position de l'apophyse épineuse par rapport aux bords latéraux du corps vertébral détecté (Fig. 6A) [4].

Nash et Moe améliorèrent la quantification de la rotation en utilisant la position des pédicules vertébraux par rapport au corps vertébral [22]. Ils ont également utilisé des radiographies PA. Les projections des pédicules sont généralement situées dans les parties latérales du corps vertébral mais avec l'augmentation de la rotation vertébrale elles se déplacent vers le centre du corps vertébral (Fig. 6B). 
Cette méthode a été améliorée par Stokes et al., en combinant la position des pédicules par rapport au centre du corps vertébral à des paramètres vertébraux connus, tels que la distance entre les pédicules et la distance entre les pédicules et le centre du corps vertébral (Figure 6C) [23].

Perdriolle et Vidal développèrent un torsiomètre pour mesurer l'angle de rotation vertébrale directement à partir d'une radiographie grandeur nature (Fig. 6D) [24]. Le gabarit de mesure était positionné sur la radiographie et aligné avec les bords latéraux du corps de la vertèbre mesurée et montrait l'angle de rotation des vertèbres à une échelle instrumentée de $5^{\circ}$ à l'aide d'une ligne passant par le centre du pédicule interne. Bien que le torsiomètre de Perdriolle soit largement utilisé en pratique clinique, sa reproductibilité et sa précision sont très limitées [25]. Plusieurs autres méthodologies furent également développées, mais tous les principes peuvent être rattachés à l'une des quatre techniques décrites ci-dessus [22].

Toutes les procédures proposées pour la quantification de la rotation axiale sur les radiographies PA sont peu fiables car les images radiographiques ne fournissent pas d'informations quantitatives ou qualitatives suffisamment précises sur les structures anatomiques nécessaires pour déterminer la rotation axiale. De plus, chaque auteur a limité son analyse à la rotation axiale, en oubliant la translation latérale des vertèbres qui est également une composante essentielle des changements au plan axial [22].

\section{MESURES « $2,5 \mathrm{D} »$}

Quelques méthodes furent développées en utilisant des clichés stéréoradiographiques, des radiographies obliques latérales et PA ou orthogonales bidimensionnelles [26] pour améliorer la précision des mesures de la rotation vertébrale axiale [27]. Les principes de ces techniques de mesure et leur mise en œuvre sont généralement les mêmes que ceux proposés par l'auteur senior de cet article. Les mesures de rotation axiale sont effectuées après reconstruction 3D des vertèbres, sur la base de l'identification des mêmes repères anatomiques des vertèbres sur les radiographies coronale et sagittale. Pour une reconstruction 3D précise et reproductible, une définition exacte des points de référence vertébraux sur des radiographies PA et latérales perpendiculaires précises est nécessaire. Par conséquent, une attention particulière à la position du patient est nécessaire pour obtenir un angle précis de $90^{\circ}$ sur les radiographies coronale et sagittale.

Les mesures de rotation les plus précises peuvent être obtenues après reconstruction 3D des vertèbres [28]. Néanmoins de nombreuses autres méthodes permettant de calculer la rotation axiale des vertèbres furent développées à l'aide de différentes formules géométriques ou de logiciels dans les systèmes d'imagerie numérique modernes [29-33]. 
MESURES «QUASI - 3D » - PLAN DE LA COURBURE MAXIMALE (PCM)

En 1994, le groupe de travail «3D Terminology of Spinal Deformity » de la SRS a reconnu la nécessité d'une méthode de diagnostic véritablement 3D de la scoliose, mais a déclaré simultanément que «la visualisation de tout ce qui est tridimensionnel est un grand défi. L'approche que nous avons adoptée tente de prendre en compte cette limitation humaine en utilisant largement les plans « auxiliaires » sur lesquels la colonne vertébrale est projetée. Ces mesures ne sont pas vraiment un angle 3D, mais cette approche utilisant des mesures « quasi 3D » représente un compromis raisonnable entre la pureté mathématique et les limites conceptuelles et pratiques »[13].

Sur la base des résultats ci-dessus, le PCM fut introduit pour la caractérisation 3D des déformations de la colonne vertébrale [34]. Le PCM est construit en utilisant le centre des deux vertèbres extrêmes et de la vertèbre apicale de la courbure. Par définition le PCM est analogue au «plan d'élection » introduit par Stagnara et al. pour évaluer radiologiquement les grandes courbes scoliotiques [35]. La définition du PCM est assez simple. Cependant, elle n'indique pas le vrai plan anatomique coronal, sagittal ou axial. Par conséquent, il est difficile de déterminer l'effet clinique et l'utilité du PCM (Figure 7) [37].

Malgré les efforts considérables et le travail acharné de chirurgiens réputés de la colonne nous pensons que l'utilisation du PCM est une abstraction ; il tente de délimiter la déformation 3D sans utiliser d'outils sophistiqués, avec lesquels il est cependant possible de représenter la projection horizontale exacte de la scoliose. Malgré les techniques qui sont constamment suggérées, l'application de ces techniques dans la pratique clinique quotidienne est compliquée. L'exposition horizontale proposée par la représentation « da Vinci »n'a pas facilité ni leur compréhension ni leur utilisation. [38].

Comme Stokes et le groupe de travail «3D Terminology of Spinal Deformity » de la SRS l'ont déclaré la visualisation du PCM n'est pas la véritable visualisation 3D mais plutôt une méthode« quasi 3D » [13].Dans ce cas, lorsqu'il est possible d'étudier la projection 3D réelle, forcer l'utilisation de plans auxiliaires non-réels (PCM) est une approche discutable.

\section{D EOS}

L'absence de visualisation 3D de la scoliose a radicalement changé en raison d'un développement complémentaire au système d'imagerie EOS 2D / 3D (EOS Imaging, Paris, France), initié par l'auteur senior de cet article [39]. Ce nouveau dispositif à rayons $\mathrm{X}$ à faible dose de rayonnement est devenu disponible pour les applications orthopédiques et repose sur la technologie de la détection des particules avec les détecteurs gazeux par G. Charpak, récompensée par le prix Nobel de physique en 1992 [40,41]. La machine EOS 2D / 3D est capable de capturer simultanément des images orthogonales, numériques et bi-planaires du patient dans une 
position debout, après la numérisation du balayage du corps entier [42]. Les radiographies PA et latérale sans distorsion et à l'échelle 1:1 créées par le système permettent une reconstruction 3D précise de la surface des vertèbres et de la colonne vertébrale grâce au logiciel spécial sterEOS développé pour accompagner le système EOS (Fig. 8). [43,44]. Après reconstruction 3D, la visualisation de la projection de la colonne vertébrale dans les trois plans anatomiques, y compris le plan transversal, est devenue possible.

La reconstruction 3D est complétée par des paramètres 3D générés automatiquement pour les vertèbres, le bassin et la colonne vertébrale. Dans le logiciel sterEOS, similaire à la biomécanique conventionnelle, les positions angulaires 3D des vertèbres sont obtenues en calculant les trois angles par la méthode des axes mobiles dans une certaine séquence (angles cardaniques ou angles de Bryant) [45]. Ceci est une définition mathématique précise. Cependant, la signification et l'utilité clinique des valeurs de rotation définies de cette manière sont discutables car il n'existe pas d'accord international général concernant les systèmes de coordonnées vertébrales et l'ordre de rotation. Dans la méthode de déplacement des axes, le calcul des trois angles dépendra de l'ordre de rotation. L'ordre de rotation dans un système de coordonnées altéré ne représente pas nécessairement le même ordre anatomique. Par conséquent, les résultats des valeurs de rotation seront différents et ne seront pas comparables [45]. Une autre erreur souvent commise par les chirurgiens orthopédistes lors de l'exploitation clinique des angles 3D de Bryant est qu'ils appellent ces angles 3D l'angle dans les plans frontal, sagittal et horizontal. Ainsi, ils confondent les angles 3D Bryant avec les angles projetés sur le plan anatomique. Cet abus de langage est trompeur, car l'angle de Bryant 3D correspond aux angles projetés dans un seul plan. En pratique clinique, seuls les angles projetés sur les plans de référence anatomiques correspondant aux angles visibles sur les clichés AP et latéraux ont une signification et seuls ces angles sont utilisés par les chirurgiens orthopédistes.

\section{VECTEUR VERTEBRAL}

Le vecteur vertébral postéro-antérieur, nouveau concept de visualisation a été introduit pour surmonter les problèmes ci-dessus, simplifier l'affichage de la projection 3D dans les plans frontal, sagittal et horizontal et permettre la caractérisation mathématique des courbures projetées (Fig. 9).) [46].

En général un vecteur est une entité mathématique caractérisée par ses points initial et terminal, sa longueur et sa direction dans l'espace, ce qui est couramment représenté par un segment orienté d'une ligne flèche.

Le point initial (A) d'un vecteur vertébral est le point médian de la ligne interpédiculaire reliant les deux centroïdes pédiculaires. L'extrémité terminale (B) est située sur la surface ventrale du corps vertébral. Par définition, le vecteur vertébral représente l'axe postéro-antérieur d'une vertèbre en étant parallèle au plateau supérieur de la vertèbre. Ainsi, la longueur du vecteur est proportionnelle à la taille du corps vertébral. Un

vecteur vertébral $\mathrm{AB}(\overrightarrow{A B})$ remplace, par définition, la vertèbre réelle tout en préservant des informations cruciales concernant sa taille, sa position et son orientation 3D [47]. 
Les vecteurs vertébraux qui représentent la colonne vertébrale complète peuvent être affichés dans un système de coordonnées d'échelle calibré individuellement et qui fournit des méthodes mathématiques simples et bien établies pour évaluer quantitativement la géométrie 3D de la colonne vertébrale (Fig. 9G). Dans ce système de coordonnées, l'axe des abscisses est une ligne droite reliant les deux centres des cavités acétabulaires. L'axe des y est perpendiculaire à l'axe des $\mathrm{x}$ dans le plan frontal et passe par le milieu de la longueur interacétabulaire, dont l'axe est situé dans le plan sagittal médian du corps. L'axe des z passe par le même point et est perpendiculaire aux axes $\mathrm{x}$ et $\mathrm{y}$.

L'échelle de ce système de coordonnées est individuelle et basée sur la distance réelle entre les deux centroïdes acétabulaires. En divisant les distances interacétabulaires en 200 unités, nous avons obtenu une échelle particulière et comparable, étant donné qu'il y a toujours 100 unités entre l'origine du système de coordonnées et les points centraux des centroïdes acétabulaires de chaque côté. Les unités ainsi obtenues sont utilisées comme unité de base pour chaque axe du système de coordonnées. Dans ce système de coordonnées, dans les trois plans, les coordonnées projetées des points initial (Ax; y; z;) et terminal (Bx; y; z;) du vecteur vertébral $\overrightarrow{A B}$ peuvent être déterminées.

Les différentes coordonnées dans les différents plans ont des significations différentes (Fig. 10A - D). Dans le plan horizontal, les coordonnées x représentent la distance par rapport à l'axe des y, les coordonnées y représentent la distance par rapport à l'axe des x et la direction du vecteur représente la rotation axiale projetée des vertèbres. La valeur projetée de la rotation vertébrale peut être calculée en utilisant les coordonnées x et y des points initiaux et terminaux des vecteurs de la vertèbre, en utilisant la fonction tangente de la trigonométrie. La valeur de rotation intervertébrale peut également être calculée et correspond à la différence de rotation axiale de deux vertèbres adjacentes. En plus de la rotation vertébrale, les déplacements latéraux des vertèbres et les projections des courbures sagittales peuvent être analysés à l'aide de ces coordonnées (Fig. 10E - F) [48].

L'affichage du vecteur vertébral a considérablement simplifié la représentation simultanée des déformations de la colonne vertébrale dans les trois plans. Son applicabilité a déjà été démontrée dans les plans frontal et sagittal pour la colonne vertébrale normale et pour la scoliose $[49,50]$.

\section{CONCLUSION}

Le vecteur vertébral est un outil amélioré pour visualiser le plan axial et mesurer la rotation dans le même plan. En pratique clinique il complète les procédures de référence actuelles pour la mesure des courbures frontales et sagittales. Bien qu'il s'agisse d'une analyse d'image par projection, depuis l'introduction des rayons X, les chirurgiens ont toujours analysé l'image projetée et sont habitués à cette méthode. Ces images de projection ne peuvent pas être évitées, même si un affichage spatial étendu et précis est également possible. Une excellente analogie en est la conception architecturale. Malgré l'existence d'une visualisation spatiale très précise, les 
bâtiments ont toujours été construits sur la base de ces plans, qui représentent les projections du bâtiment dans les trois plans. La construction d'un édifice basé uniquement sur une image d'ordinateur 3D est impossible. Il en va de même pour la scoliose, il est également nécessaire de connaître les trois projections dans les trois plans. Il ne suffit pas d'avoir uniquement des images en deux plans, la scoliose étant une déformation en 3D.

En utilisant le vecteur vertébral PA, les chirurgiens sont capables d'analyser la troisième dimension de la scoliose sans devoir appliquer d'autres plans virtuels. L'approche est simple. Cette méthode de visualisation graphique conviviale facilite considérablement la compréhension de la nature 3D de la scoliose et aide à suivre les résultats du traitement conservateur [51] et du traitement chirurgical [52]. Ces résultats sont suffisants pour permettre une analyse visuelle et fournir des informations cliniques significatives dans les trois plans anatomiques. En outre, cette approche de visualisation représente un compromis raisonnable entre la pureté mathématique et l'utilisation pratique.

\section{Déclarations de conflit d'intérêts :}

T.S.I. Conflit d'intérêt : Aucun

F.L. Conflit d'intérêt : Aucun

J.F.D. Consultations occasionnelles pour MEDTRONIC.

\section{Financement}

Aucun

\section{Contributions des auteurs}

TSI : conception de l'étude, plan méthodologique, rédaction de l'article

$\mathrm{FL}$ : révision critique du contenu d'importance intellectuelle

JFD : révision critique et approbation finale de la version soumise pour publication 


\section{References}

1. Magny M. Mémoire sur le Rakitis ou Maladie de la Colonne vertebrale. Paris: Chez Méquignon l'aîné, Libraire, rue des Cordeliers, vis-à-vis l'Eglise de Saint-Côme; MDCCLXXX.

2. Adams W. Lectures Pathology and Treatment Lateral and Other Forms curvature of the Spine. $2^{\text {nd }}$ ed. London: J. \& A. Churchill New Burlington street; 1882.

3. Röntgen W. On a new kind of rays. Nature 1895-1896;53:274-26.

4. Cobb JR. Outline for the study of scoliosis. In The American Academy of Orthopedic Surgeons. Instructional Course Lectures. Ann Arbor: JW. Edwards; 1948;5: 261-75.

5. Diebo BG, Varghese JJ, Lafage R, Schwab FJ, Lafage V. Sagittal alignment of the spine: What do you need to know? Clin Neurol Neurosurg 2015;139:295-301.

6. Roussouly P, Nnadi C. Sagittal plane deformity: an overview of interpretation and management. Eur Spine J 2010;19:1824-36.

7. Lenke LG, Betz RR, Harms J, et al. Adolescent idiopathic scoliosis: a new classification to determine extent of spinal arthrodesis. J Bone Joint Surg Am 2001;83-A: 1169-81.

8. Graf H, Hecquet J, Dubousset J. 3-dimensional approach to spinal deformities. Application to the study of the prognosis of pediatric scoliosis. Rev Chir Orthop 1983;69:407-16. [in French]

9. Cotrel Y, Dubousset J. Nouvelle technique d'ostéosynthese rachidienne segmentaire par voie postérieure. Rev Chir Orthop 1984;70: 489-94.

10. Dubousset J, Herring JA, Shufflebarger H. The Crankshaft Phenomenon. J Pediatr Orthop 1989;9:541-50.

11. de Jonge T, Dubousset JF, Illés T. Hyperrotatory paradoxic kyphosis. Spine 2002;27: 393-8. 
12. Cotrel Y, Dubousset J, Guillaumat M. New universal instrumentation in spinal surgery. Clin Orthop Relat Res 1988;227:10-23.

13. Stokes IA. Three-dimensional terminology of spinal deformity. A report presented to the Scoliosis Research Society by the Scoliosis Research Society Working Group on 3-D terminology of spinal deformity. Spine 1994;19:236-48.

14. Aaro S, Dahlborn M. Estimation of vertebral rotation and the spinal and rib cage deformity in scoliosis by computer tomography. Spine 1981;6:460-7.

15. Ho E, Upadhyay S, Chan F, Hsu L, Leong J. New methods of measuring vertebral rotation from computed tomographic scans: an intraobserver and interobserver study on girls with scoliosis. Spine 1993;18:1173-7.

16. Krismer M, Sterzinger W, Christian H, Frischhut B, Bauer R. Axial rotation measurement of scoliotic vertebrae by means of computed tomography scans. Spine 1996;21:576-81.

17. Vrtovec T, Likar B, Pernus F. Automated curved planar reformation of 3D spine images. Phys Med Biol 2005;50:4527-40.

18. Birchall D, Hughes D, Hindle J, Robinson L, Williamson J. Measurement of vertebral rotation in adolescent idiopathic scoliosis using three-dimensional magnetic resonance imaging. Spine 1997;22:2403-7.

19. Vrtovec T, Ourselin S, Lavier G, Likar B, Pernus F. Automated generation of curved planar reformations from MR images of the spine. Phys Med Biol 2007;52:2865-78.

20. Yazici M, Acaroglu ER, Alanay A, Deviren V, Cila A, Surat A. Measurement of vertebral rotation in standing versus supine position in adolescent idiopathic scoliosis. J Pediatr Orthop $2001 ; 21: 252-6$.

21. Vrtovec T, Franjo Pernus F, Likar B. A review of methods for quantitative evaluation of axial 
vertebral rotation. Eur Spine J 2009;18:1079-90.

22. Nash Jr CL, Moe JH. A study of vertebral rotation. J Bone Joint Surg Am1969;51-A:223-9.

23. Stokes IA, Bigalow LC, Moreland MS. Measurement of axial rotation of vertebrae in scoliosis. Spine 1986;11:213-8.

24. Perdriolle R, Vidal J. A study of scoliotic curve. The importance of extension and vertebral rotation [in French]. Rev Chir Orthop Reparatrice Appar Mot 1981;67:25-34.

25. Omeroglu H, Ozekin O, Bicimoglu A. Measurement of vertebral rotation in idiopathic scoliosis using the Perdriolle torsionmeter: a clinical study on intraobserver and interobserver error. Eur Spine J 1996;5:167-71.

26. Pearcy M, Tibrewal S. Axial rotation and lateral bending in the normal lumbar spine measured by three-dimensional radiography. Spine 1984;9: 582-7.

27. Labelle H, Dansereau J, Bellefleur C, Jéquier J. Variability of geometric measurements from three-dimensional reconstructions of scoliotic spines and rib cages. Eur Spine J 1995;4: 8894.

28. Gille O, Champain N, Benchikh-El-Fegoun A, Vital JM, Skalli W. Reliability of 3D reconstruction of the spine of mild scoliotic patients. Spine 2007;32:568-73.

29. Chi WM, Cheng CW, Yeh WC, et al. Vertebral axial rotation measurement method. Comput Methods Programs Biomed 2006;81:8-17.

30. Eijgcnraam SM, Boselie TF, Sieben JM, et al. Development and assessment of a digital X-ray software tool to determine vertebral rotation in adolescent idiopathic scoliosis. Spine $\mathbf{J}$ 2017;17:260-5.

31. Hecquet J, Legaye J, Duval-Beaupère G. Access to a threedimensional measure of vertebral axial rotation. Eur Spine J. 1998;7:206-11. 
32. Pinheiro AP, Tanure MC, Oliveira AS. Validity and reliability of a computer method to estimate vertebral axial rotation from digital radiographs. Eur Spine J 2010;19:415-20.

33. Sullivan TB, Bastrom T, Reighard F, Jeffords M, Newton PO. A novel method for estimating three-dimensional apical vertebral rotation using two-dimensional coronal Cobb angle and thoracic kyphosis. Spine Deformity 2017;5: 244-9.

34. Aubin C-E, Lobeau D, Labelle H, Maquinghen-Godillon AP, LeBlanc R, Dansereau J. Planes of maximum deformity in the scoliotic spine. In: IAF Stokes (ed) Research into spinal deformities 2. Amsterdam: IOS Press;1999. p 45-8.

35. Stagnara P, Queneau P. Scolioses évolutives en période de croissance: aspects cliniques, radiologiques, propositions thérapeutiques. Rev Chir Orthop 1953;39:378-449.

36. Labelle H, Aubin C-E, Jackson R, Lenke L, Newton P, Parent S. Seeing the spine in 3D: How will it change what we do? J Pediatr Orthop 2011;31(Suppl.1): S37-S45.

37. Villemure I, Aubin C-E, Grimard G, Dansereau J, Labelle H. Progression of vertebral and spinal three-dimensional deformities in adolescent idiopathic scoliosis: A longitudinal study. Spine 2001;26:2244-50.

38. Sangole AP, Aubin CE, Labelle H, Stokes IA, Lenke LG, Jackson R, Newton P. Threedimensional classification of thoracic scoliotic curves. Spine 2009;34:91-9.

39. Dubousset J. Charpak G, Dorion I, et al. Le system EOS. Nouvelle imagerie osteo-articulaire basse dose en position debout. E-mémoire de l'Académie Nationale de Chirurgie 2005;4:227.

40. Kalifa G, Charpak G, Maccia C et al. Evaluation of a new low-dose digital x-ray device: first dosimetric and clinical result in children. Pediatr Radiol 1998;28:557-61.

41. http://nobelprize.org/nobel_prizes/physics/laureates/1992/index.html 
42. Dubousset J. Charpak G, Dorion I et al. A new 2D and 3D imaging approach to musculoskeletal physiology and pathology with low-dose radiation and the standing position: the EOS sytem. Bull Acad Natl Med 2005;189:287-97.

43. Mitulescu A, Skalli W, Mitton D, De Guise J. Three-dimensional surface rendering reconstruction of scoliotic vertebrae using a non stereo-corresponding points technique Eur Spine J 2002;11:344-52.

44. Le Bras A, Laporte S, Mitton D, de Guise JA, Skalli W. 3 D detailed reconstruction of vertebrae with low dose digital stereoradiography. Stud Health Technol Inform 2002;91:28690.

45. Skalli W, Lavaste F, Descrimes JL. Quantification of three-dimensional vertebral rotations in scoliosis: what are the true values? Spine 1995;20:546-53.

46. Illés T, Tunyogi-Csapó M, Somoskeöy Sz. Breakthrough in three-dimensional scoliosis diagnosis - significance of horizontal plane view and vertebra vectors. Eur Spine J 2011;20:135-43.

47. Illés T. Comparaison entre la mesure classique bidimensionnelle de la scoliose et sa mesure tridimensionnelle par les vecteurs vertébraux. Avantages dans le pronostic et l'appréciation des résultats. Bull Acad Nat Medecine 2011;195:629-43.

48. Illés TS, Burkus M, Somoskeöy S, Lauer F, Lavaste F, Dubousset JF. The horizontal plane appearances of scoliosis: what information can be obtained from top-view images? Int Orthop. 2017;41:2303-11.

49. Somoskeöy S, Tunyogi-Csapó M, Bogyó C, Illés T. Clinical validation of coronal and sagittal spinal curve measurements based on three-dimensional vertebra vector parameters. Spine J. 2012;12:960-8. 
50. Illés T, Somoskeöy S. Comparison of scoliosis measurements based on three-dimensional vertebra vectors and conventional two-dimensional measurements: advantages in evaluation of prognosis and surgical results. Eur Spine J 2013;22:1255-63.

51. Ilharreborde B, Sbag G, Skalli W, Mazda K. Adolescent idiopathic scoliosis treated with posteromedial translation: radiologic evaluation with a 3D low-dose system. Eur Spine J 2013;22:2382-91.

52. de Mauroy JC, Journe A, Gagaliano F, Lecante C, Barral F, Pourret S. The new Lyon ARTbrace versus the historical Lyon brace: a prospective case series of 148 consecutive scoliosis with short time results after 1 year compared with a historical retrospective case series of 100 consecutive scoliosis; SOSORT award 2015 winner. Scoliosis 2015;10:26. https://doi.org/10.1186/s13013-015-0047-6) 
FIGURES

Fig. 1 : M. Magny: Mémoire sur la Rakitis ou maladie de la colonne vertébrale [1]

C'est sans doute la première représentation de la projection de scoliose dans le plan axial.

Fig. 2 : Plan axial d'une représentation de scoliose en vue de dessus, selon Adams [2]

Une représentation étonnamment précise de la rotation axiale des vertèbres (A), de la structure d'un thorax normal (B) et de la déformation thoracique pendant la formation de la gibbosité après la rotation axiale des vertèbres $(\mathrm{C})$.

Fig. 3 : La première visualisation de la colonne vertébrale en utilisant la technologie informatique [8]

Des radiographies PA et latérales debout ont été utilisées pour marquer la limite postérieure du plateau sacré et le centre de chaque plateau vertébral sur la vue PA, tandis que le bord postérieur de chaque plateau vertébral était marqué sur une radiographie latérale (points bleus sur le schéma image, A). La rotation de chaque vertèbre a également été mesurée sur une vue PA en utilisant la technique de Perdriolle. Sept points ont été utilisés pour chaque vertèbre.

Sur la base des points obtenus, l'ordinateur a alors directement produit une reconstruction tridimensionnelle de la colonne vertébrale, montrant une image des courbures dans les plans frontal, sagittal et axial, sur lesquelles chaque droite représente une vertèbre. La longueur de chacune des petites lignes varie en fonction de la hauteur de la vertèbre et de son inclinaison sur le plan horizontal. (B) Un affichage idéalisé du plan axial de la colonne vertébrale était également disponible à l'aide de l'ordinateur (C)

Fig. 4 : Le phénomène de vilebrequin [10]

Images radiographiques préopératoires (A) et postopératoires tardives (C) PA du même patient.

Les vues supérieures préopératoires (B) et postopératoires tardives (D) démontrent qu'en raison de la rotation extrême $\left(>90^{\circ}\right)$ des vertèbres apicales (Th10), la position de la masse de fusion (noire) s'est progressivement déplacée en avant vers les corps vertébraux. Ainsi, la croissance des corps vertébraux produit non seulement une 
angulation et une rotation supplémentaires de la colonne vertébrale mais également une cyphose progressive apparente.

Fig. 5 : Différentes vertèbres ayant subi une rotation chez un patient atteint de scoliose, vues par tomographie.

Fig. 6 : Méthodes d'évaluation quantitative de la rotation vertébrale axiale [21]

Méthode Cobb (A) ; Méthode Nash et Moe (B) ; Méthode Stokes (C) ; Méthode de Perdriolle (D).

Fig. 7 : PCM et son affichage en vue de dessus en utilisant la vue et la représentation «da Vinci» [36]

Images radiographiques latérales (A) et PA (B) d'un patient atteint d'une scoliose thoracique majeure. La vue d'en haut des vertèbres apicales est représentée sur un graphique circulaire (C). Reconstruction 3D de la même scoliose (D).

Les PCM thoracique supérieur (bleu), thoracique principal (vert), thoraco-lombaire / lombaire (orange) dans le plan frontal (E) et vue dans le plan axial (F).

La projection « da Vinci » $(\mathrm{G})$ est une représentation schématique des plans PCM et de la sévérité des différents segments de la colonne vertébrale.

La représentation « da Vinci » $(\mathrm{H})$ des mêmes courbures dans laquelle les PCM sont remplacés par une flèche se projetant du centre vers le sommet du PCM.

La longueur de la flèche est proportionnelle à la courbure; sa projection horizontale reflète l'ampleur de la déformation coronale et sa projection verticale reflète la composante sagittale de la déformation. De plus, la rotation du corps vertébral est représentée par un affichage graphique simplifié de chaque vertèbre apicale avec l'apophyse spinale, indiquant la direction et la quantité de rotation dans le plan transversal.

Fig. 8 : Images EOS 2D / 3D d'une scoliose sévère

Reconstruction 3D sur radiographie PA (A) et radiographie latérale (B). Vue d'en haut 3D (C).

Fig. 9 : Détermination du vecteur vertébral et du système de coordonnées [45] 
Vertèbres 3D reconstruites en surface en vue frontale (A), sagittale (B) et axiale (C) en relation et positionnement du vecteur vertébral (flèche magenta).

Vues frontale $(\mathrm{D})$, sagittale $(\mathrm{E})$ et axiale $(\mathrm{F})$ du contour des vertèbres, points de référence colorés pour le repère vertébral.

Vue en plan horizontal du système de coordonnées avec une vertèbre (mode contour) et son vecteur vertébral (G).

Les coordonnées de chaque point vectoriel peuvent être déterminées en utilisant une algèbre vectorielle de base.

Les coordonnées du vecteur $\mathrm{AB}(\overrightarrow{A B})$ sont $\left(\mathrm{A}_{\mathrm{x}} ; \mathrm{A}_{\mathrm{y}}\right)$ du point initial $\mathrm{A}$ et $\left(\mathrm{B}_{\mathrm{x}} ; \mathrm{B}_{\mathrm{y}}\right)$ du point terminal $\mathrm{B}$. Par conséquent, les coordonnées sont les suivantes : $\overrightarrow{A B}\left(B_{x}-A_{x} ; B_{y}-A_{y}\right)$.

La longueur projetée (d) de $\overrightarrow{A B}$ dans le plan axial $: d_{\overrightarrow{A B}}=\sqrt{\left(A_{y}-B_{y}\right)^{2}+\left(A_{x}-B_{x}\right)^{2}}$.

La rotation axiale (l'angle vectoriel $\square$ par rapport à l'axe y) est calculée à l'aide de la fonction tangente: $\operatorname{tg} a=$ $\frac{(B x-A x)}{(B y-A y)}$.

\section{Fig. 10 : EOS 2D/3D et visualisation d'une scoliose sévère par des vecteurs vertébraux}

Reconstruction 3D sur une radiographie PA (A). Remplacement des vertèbres par des vecteurs vertébraux (B). Avec la visualisation par vecteur vertébral dans le plan frontal, seules les petites flèches représentant les points terminaux des vecteurs sont visibles si les vertèbres ne présentent pas de rotation axiale (T7 et T8). En raison de la rotation vertébrale la projection visible des vecteurs augmente, progressant de sorte que les vecteurs semblent être de pleine longueur. Cela indique une rotation de presque $90^{\circ}$ ( $\mathrm{L} 1$ et L2). La décompensation du haut du corps peut également être déterminée à partir d'une distance entre $\mathrm{T} 1$ et l'axe z. La magnitude de la courbure frontale est directement proportionnelle aux coordonnées $\mathrm{x}$ du vecteur vertébral.

Radiographie latérale avec reconstruction 3D (C) et visualisation vectorielle des vertèbres (D). Dans le plan sagittal la plupart des vecteurs sont visibles en pleine longueur en montrant clairement les courbures sagittales de la colonne vertébrale. Au fur et à mesure que la rotation axiale augmente, les longueurs vectorielles projetées diminuent de manière continue alors que dans les cas de rotation de $90^{\circ}$, seules les pointes de flèche sont visibles ( $\mathrm{L} 1$ et $\mathrm{L} 2$ ).

Reconstruction 3D en vue d'en haut (E) et visualisation du vecteur vertébral(F). Dans le plan axial la rotation vertébrale n'affecte pas la longueur projetée des vecteurs ; ainsi l'angle en rapport avec l'axe y montre les rotations vertébrales axiales projetées. Les coordonnées $\mathrm{x}$ mesurées dans le plan axial sont directement proportionnelles au déplacement latéral des vertèbres [42]. 


\section{FIGURE}

$-1$

wer D,U PEAN rar

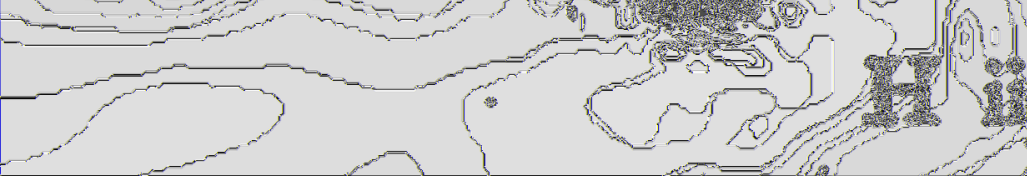



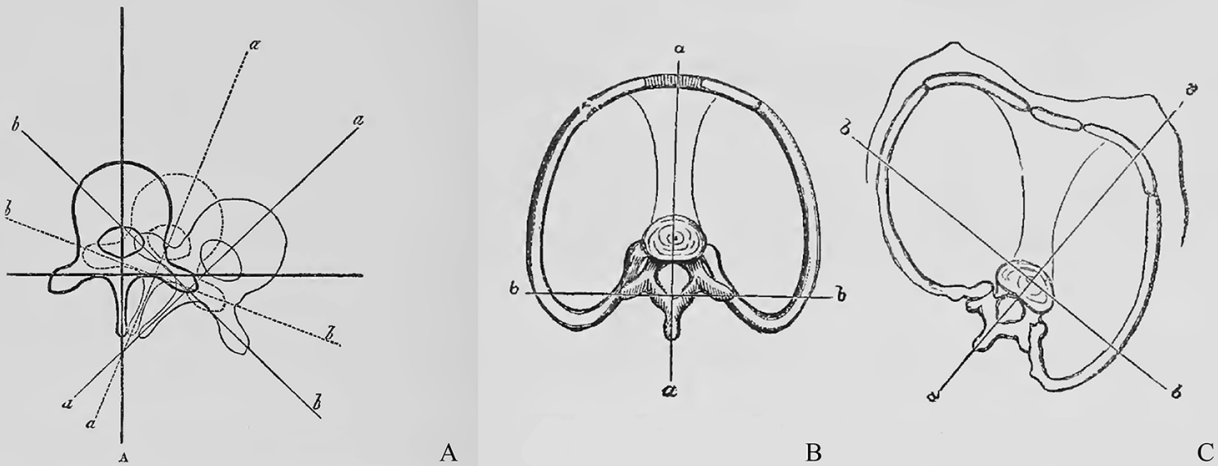

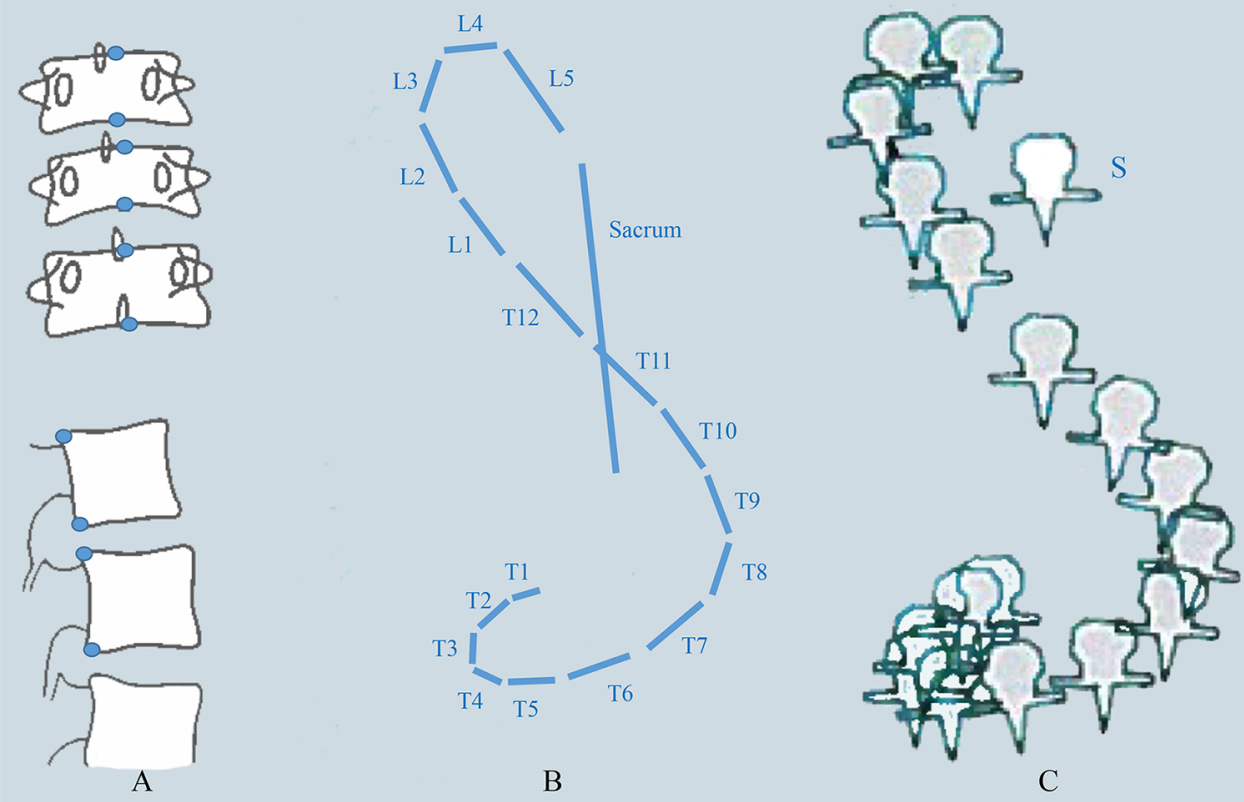


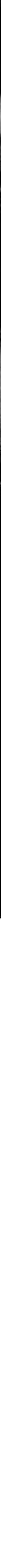




$$
1
$$

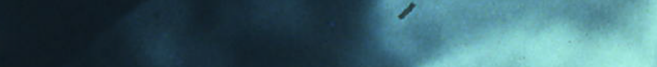

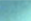



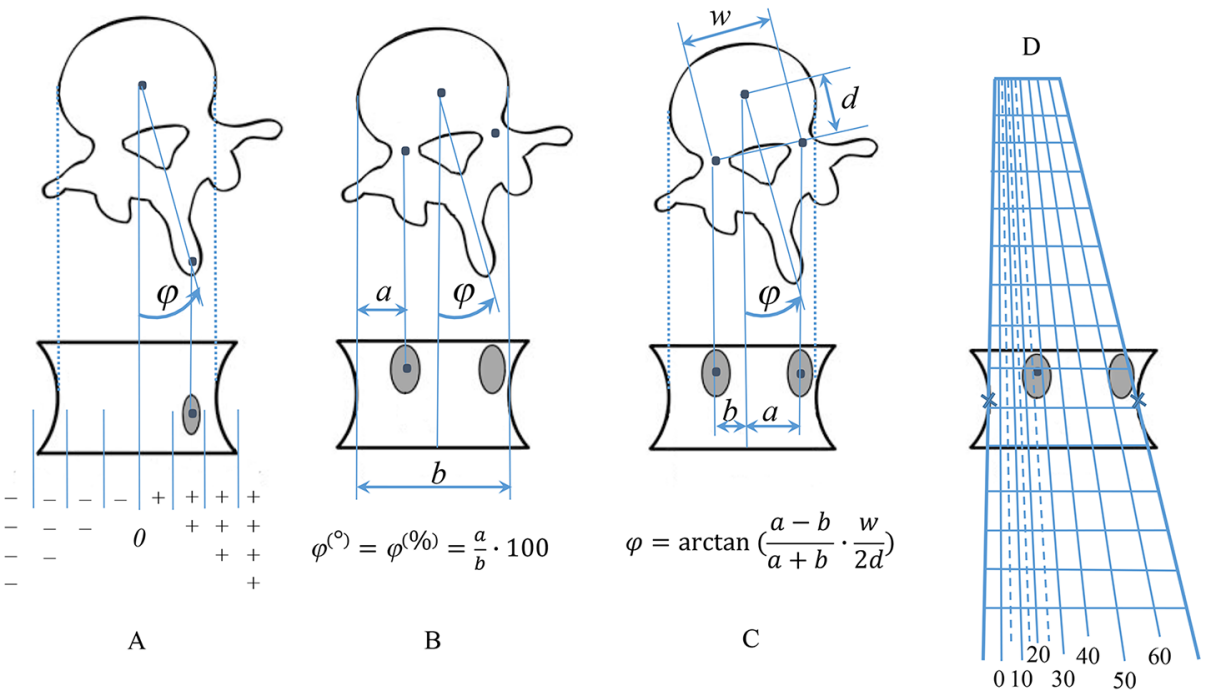


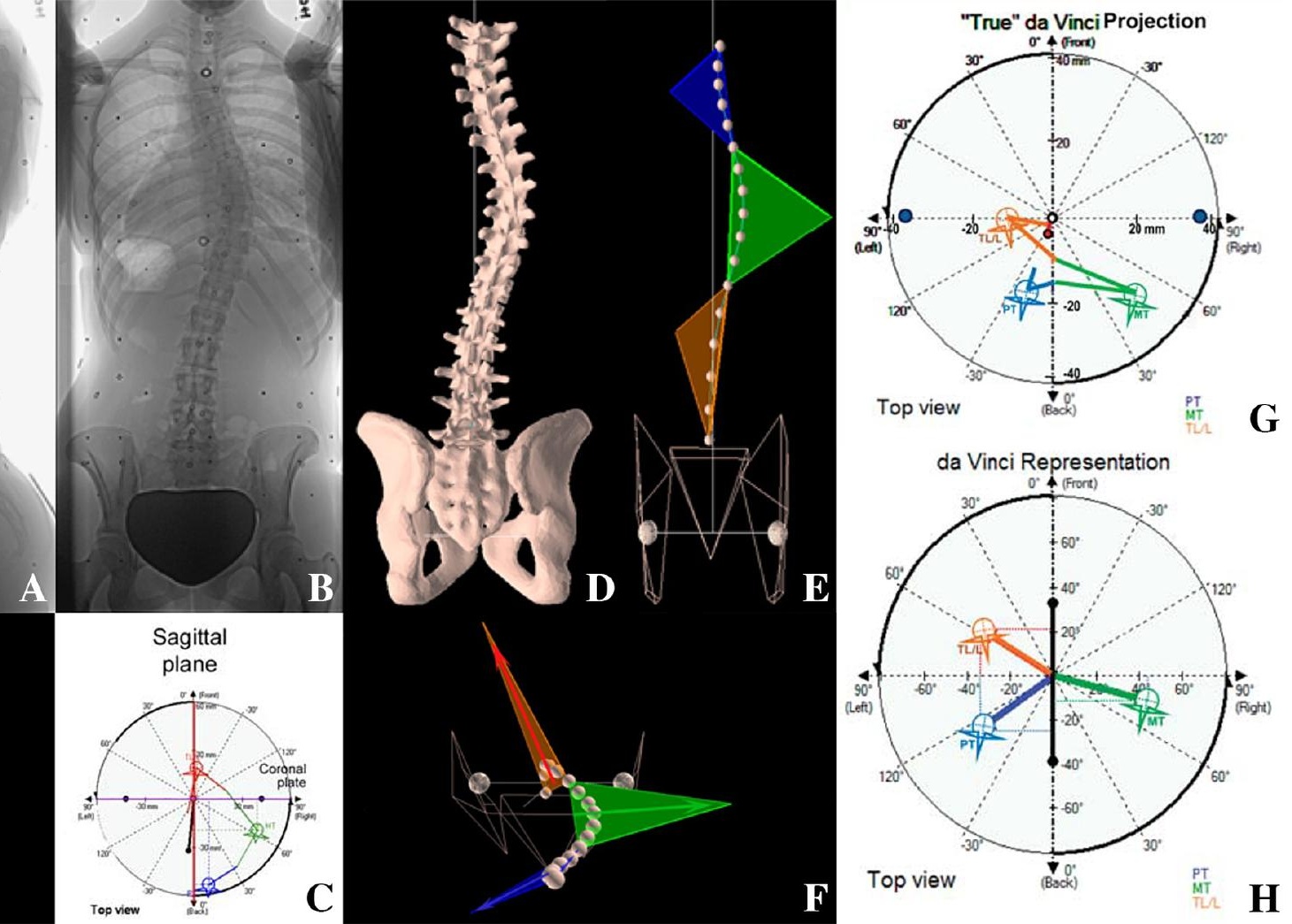




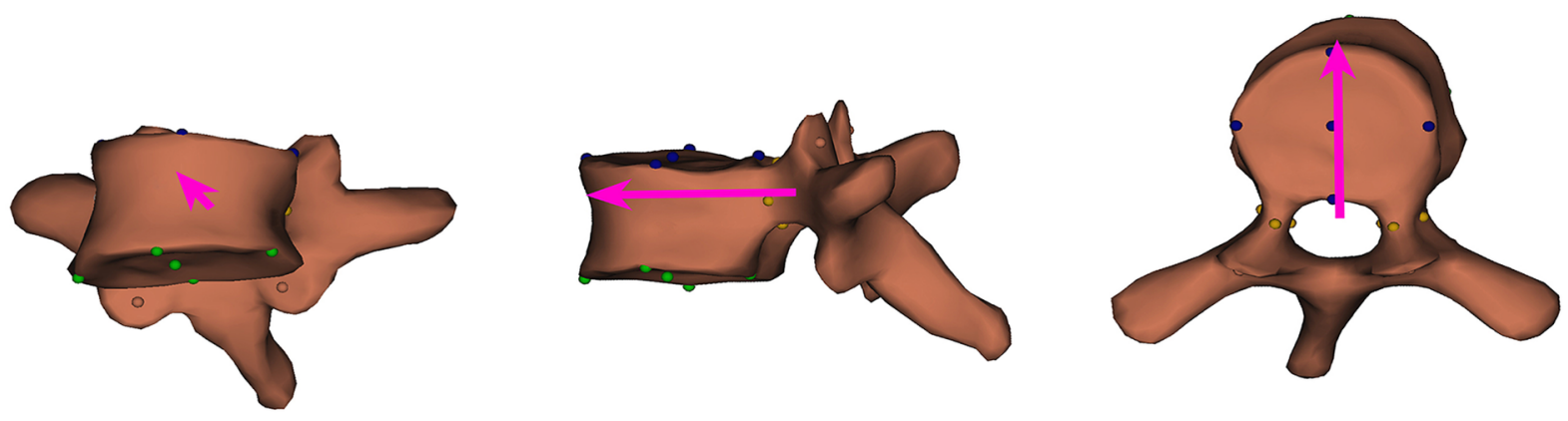

A

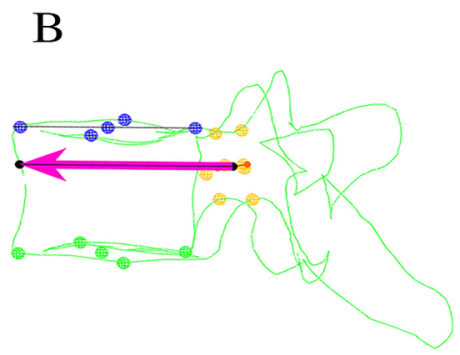

D

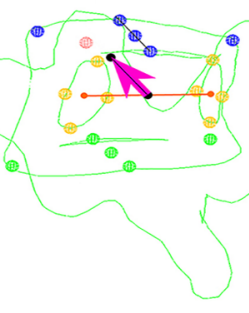

E
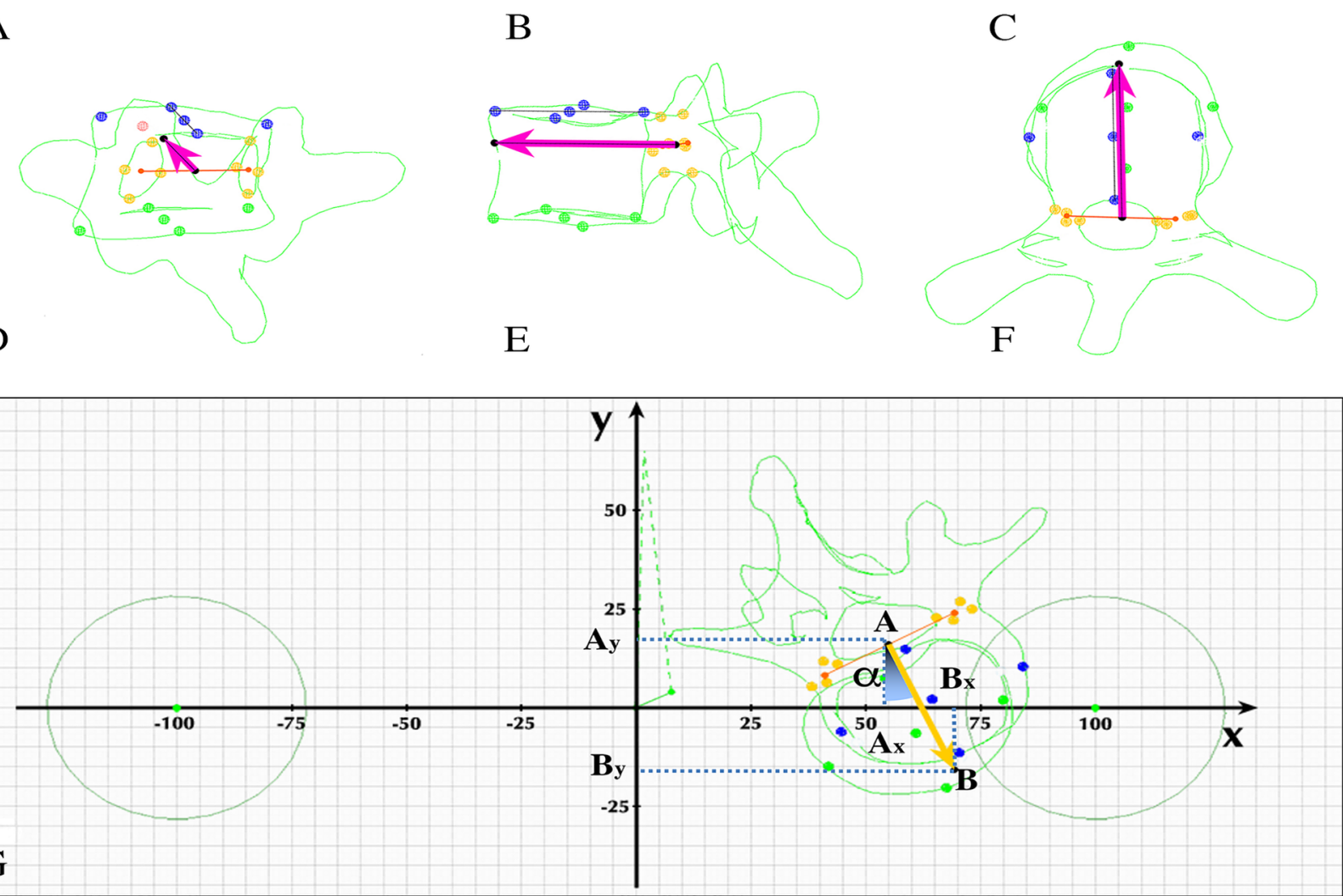


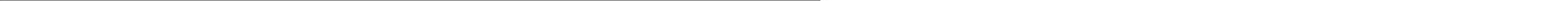

